\title{
Biomarker expression in normal fimbriae: Comparison of high- and low-grade serous ovarian carcinoma
}

\author{
ZHANG XUYIN*, DING JINGXIN*, TAO XIANG, JIA LUOQI and HUA KEQIN \\ Department of Obstetrics and Gynecology, Obstetrics and Gynecology Hospital, \\ Fudan University, Shanghai 200090, P.R. China
}

Received May 15, 2012; Accepted August 13, 2012

DOI: $10.3892 / \mathrm{ol} .2012 .877$

\begin{abstract}
The objective of this study was to assess the difference in fimbriae of high- and low-grade ovarian serous carcinoma (OSC). The fimbriae of normal appearance [without serous tubal intraepithelial carcinoma (STIC)] from 28 patients with high-grade OSCs and 24 patients with low-grade OSCs were assessed for the expression of 6 markers [E-cadherin, matrix metalloproteinase-2 (MMP-2), phospho-AKT (pAKT), cyclooxygenase-2 (COX-2), vascular endothelial growth factor (VEGF) and p53] using immunohistochemistry. Sectioning and extensively examining the fimbria (SEE-FIM) was performed to exclude fimbrial involvement for all the cases. The immunostaining levels of pAKT and COX-2 were significantly higher in the fimbriae of normal appearance from high-grade OSCs compared with low-grade OSCs (61 vs. 8\% and 71 vs. $21 \%$; $\mathrm{P}=0.005$ and 0.007 , respectively). The immunostaining of E-cadherin was significantly higher in the fimbriae of low-grade OSCs compared with high-grade OSCs ( 83 vs. $21 \%$; $\mathrm{P}=0.003$ ). The remaining 3 markers (MMP-2, VEGF and p53) had similar expression in low- and high-grade OSCs (21 vs. $13 \%$; 25 vs. $21 \%$; and 14 vs. $8 \%$; $\mathrm{P}=0.78,0.86$ and 0.82 , respectively). Our results suggest marked biological differences in the behavior of the fimbriae in high- and lowgrade OSCs and indicate that proliferation, cell adhesion and the inflammatory microenvironment of fimbriae in high-grade OSCs without STIC had changed prior to p53 mutation.
\end{abstract}

\section{Introduction}

Epithelial ovarian cancer is the most common cause of mortality from gynecological malignancy, and most ovarian

Correspondence to: Dr Hua Keqin, Department of Obstetrics and Gynecology, Obstetrics and Gynecology Hospital, Fudan University, 128 Shen Yang Road, Shanghai 200090, P.R. China

E-mail: huakeqin@126.com

*Contributed equally

Key words: high-grade serous, low-grade serous, fimbria, phospho-AKT, matrix metalloproteinase-2, E-cadherin, vascular endothelial growth factor, cyclooxygenase-2, p53 carcinomas are of the serous type. Serous carcinomas can be further subclassified as high- or low-grade, based on histological features (1). The group termed 'atypical proliferative serous tumor (APST)' behave in a benign fashion and a second, smaller group designated 'micropapillary serous carcinoma (MPSC)' (also termed 'noninvasive low-grade serous carcinoma') behave in a similar manner to low-grade malignant tumors (2). Moreover, the latter subset has been found to be closely associated with invasive low-grade serous carcinoma (LGSC) and the authors proposed that MPSC was the immediate precursor of LGSC. LGSC is a distinct entity that differs from high-grade serous carcinoma (HGSC) in several ways, for example showing specific mutations in genes such as BRAF and KRAS (2).

Low-grade invasive tumours, which have a better prognosis, are regarded as ovarian-derived. However, some findings make a strong argument that the ovarian epithelial inclusions with a tubal phenotype are likely derived from a Fallopian tube through an intraovarian endosalpingiosis rather than through Müllerian metaplasia from the ovarian surface epithelium (3). It is possible that their site of origin will be re-evaluated in the future. Nevertheless, it is clear that serous borderline cancers are not precursor lesions for the majority of high-grade serous ovarian cancer, as they have a distinct range of mutational events (4).

There is emerging and compelling evidence that a number of high-grade ovarian serous carcinomas (OSCs) originate from the epithelium of the distal fimbrial portion of the Fallopian tube. The Fallopian tube mucosa was thus suggested to be a strong candidate for the primary source of pelvic (ovarian, tubal or peritoneal) serous carcinoma. Serous tubal intraepithelial carcinoma (STIC) has been implicated in the origins of not only this group, but also serous carcinomas and primary peritoneal carcinomas. It has been proposed that the earliest neoplastic change begins in secretory-type cells (5). Further evidence supporting the proposal that STICs are precursors comes from the identification of STICs in females without ovarian cancer, as well as the presence of identical p53 mutations in STICs and concomitant ovarian HGSCs, indicating a clonal relationship (6).

Most epithelial malignancies arise through a sequence of genotypic events leading to malignant phenotypes, regardless of where they occur. Notably, Kindelberger et al (6) reported that $47 \%$ of tumors classified as OSC coexisted with STIC. 
Studies dealing with biomarkers, including markers of inflammation, micronenvironment, proliferation and invasion, in normal fimbriae (without STIC) of high- and low-grade OSC are scarce. In the present study, we detected the expression of 5 markers [E-cadherin, matrix metalloproteinase-2 (MMP-2), phospho-AKT (pAKT), cyclooxygenase-2 (COX-2), vascular endothelial growth factor (VEGF) and p53] in the normal-appearing fimbriae with high- and low-grade OSCs by immunohistochemistry. The aim of this study was to assess the difference in fimbriae of low- and high-grade OSC without STIC. We investigated whether the invasion, proliferation, inflammatory microenvironment, cell adhesion and angiogenesis of fimbriae of high-grade OSCs without STIC changed prior to 553 mutation.

\section{Materials and methods}

Case selection. Slides of fimbria tissue, all resected between January 2008 and December 2010 from a total of 52 patients, were used in this study. All were obtained from the archived files of the pathology department in the Obstetrics and Gynecology Hospital of Fudan University (Shanghai, China), following approval from its institutional review board. Patient consent was received either from the patient or the patient's family. The group comprised 28 HGSCc and 24 LGSCs that had previously been categorized as being of ovarian origin based on conventional criteria (1). All cases were reviewed and classified independently as low- or high-grade by two gynecological pathologists using histological criteria described previously (1). Sectioning and extensively examining the fimbria (SEE-FIM) was performed and the 2 pathologists reviewed the hematoxylin and eosin-stained sections from each specimen to exclude fimbria involvement for all the cases independently.

Marker selection and immunohistochemistry. Immunohistochemical (IHC) staining for all 6 markers (E-cadherin, cell adhesion; MMP-2, invasion; pAKT, proliferation; COX-2, inflammatory microenvironment; VEGF, angiogenesis; and p53) was performed following standard IHC procedures. Details of antibodies used and staining conditions are provided in Table I.

The rabbit monoclonal antibodies against E-cadherin, pAKT and COX-2 were purchased from Cell Signaling Technology (Denvers, MA, USA) and the goat polyclonal antibodies against MMP-2, VEGF and p53 were purchased from R\&D Systems (Minneapolis, MN, USA) and Dako (Carpinteria, CA, USA), all of which were used as primary antibodies. The dilutions are listed in Table I. Formalin-fixed, paraffin-embedded specimens were sliced into 5- $\mu \mathrm{m}$ sections, placed on glass slides and routine deparaffinization and rehydration procedures were performed. For antigen retrieval, the slides were heated at $98^{\circ} \mathrm{C}$ in an EDTA buffer ( $\mathrm{pH}$ 9.0) for a total of $45 \mathrm{~min}$ and cooled naturally to the room temperature. The cooled slides were rinsed with distilled water and transferred to phosphate-buffered saline (PBS). After the slides were rinsed in PBS, the endogenous peroxidase activity was quenched by incubating the sections for $15 \mathrm{~min}$ with $0.3 \%$ $\mathrm{H}_{2} \mathrm{O}_{2}$ in absolute methanol. The slides were subsequently dehydrated in PBS and incubated with blocking serum (10\% nonimmune goat serum) for $30 \mathrm{~min}$ at room temperature.
Table I. Antibodies.

\begin{tabular}{lllll}
\hline Marker name & Specific & Supplier & Dilution & Catalog no. \\
\hline pAKT & Rabbit & CST & $1: 1,000$ & 4060 \\
E-cadherin & Rabbit & CST & $1: 1,000$ & 3195 \\
COX-2 & Rabbit & CST & $1: 1,000$ & 4842 \\
MMP-2 & Goat & R\&D & $1: 200$ & AF902 \\
VEGF & Goat & R\&D & $1: 200$ & AB-293-NA \\
p53 & Goat & Dako & $1: 200$ & M 7001
\end{tabular}

pAKT, phospho-AKT; COX-2, cyclooxygenase-2; MMP-2, matrix metalloproteinase-2; VEGF, vascular endothelial growth factor; CST, Cell Signaling Technology, Danvers, MA, USA; R\&D, R\&D Systems, Minneapolis, MN, USA; Dako, Carpinteria, CA, USA.

After slides were rinsed, they were incubated with biotinylated secondary antibody detection reagent at room tempetature for $30 \mathrm{~min}$. Following incubation with the antibodies, the sections were washed with PBS 3 times and incubated with an avidinbiotinylated horseradish peroxidase macromolecular complex for $10 \mathrm{~min}$, according to the manufacturer's instructions. The bound antibody complexes were stained for 3-5 min or until appropriate for microscopic examination with diaminobenzidine and then counterstained with hematoxylin (for $30 \mathrm{sec}$ ) and mounted. Briefly, images were captured with the microscope (Olympus BX51, Olympus, Tokyo, Japan) fitted with a digital camera (Olympus DP70, Olympus).

All IHC stainings were performed independently by the same pathologists with experience of this technique.

Evaluation and scoring of immunohistochemically analyzed tissue sections. All immunostained tissue sections were evaluated and scored by a board-certified pathologist with 10 years' experience of IHC techniques both in research and diagnostic pathology who was blinded to any clinical or pathological information about the sections. For each case, 1,000 cells were assessed in 3 or 4 different fields at a magnification of $x 400$. Expression scores were assigned semiquantitatively according to the percentage of cells stained $(1,<25 \% ; 2,25 \%-75 \% ; 3$, $>75 \%$ ) and the intensity of the staining (1, weak; 2 , moderate; 3 , strong). The two scores were then multiplied. When $<25 \%$ of the cells were stained, the intensity of the stain was weak; when the product of the 2 scores was $\leq 3$, the expression was categorized as negative. When $\geq 25 \%$ of the cells were stained, the intensity of the stain was moderate or strong; when the product of the 2 scores was $\geq 4$, the expression was categorized as positive.

Statistical analysis. Fisher's exact test was used to compare the expression of each protein in fimbrial epithelial cells of high- and low-grade OSCs. $\mathrm{P}<0.05$ was considered to indicate a statistically significant result.

\section{Results}

The immunohistochemical analysis results are summarized in Table II. Three of the 6 markers (pAKT, E-cadherin and COX-2) showed significant differences between the fimbriae of 


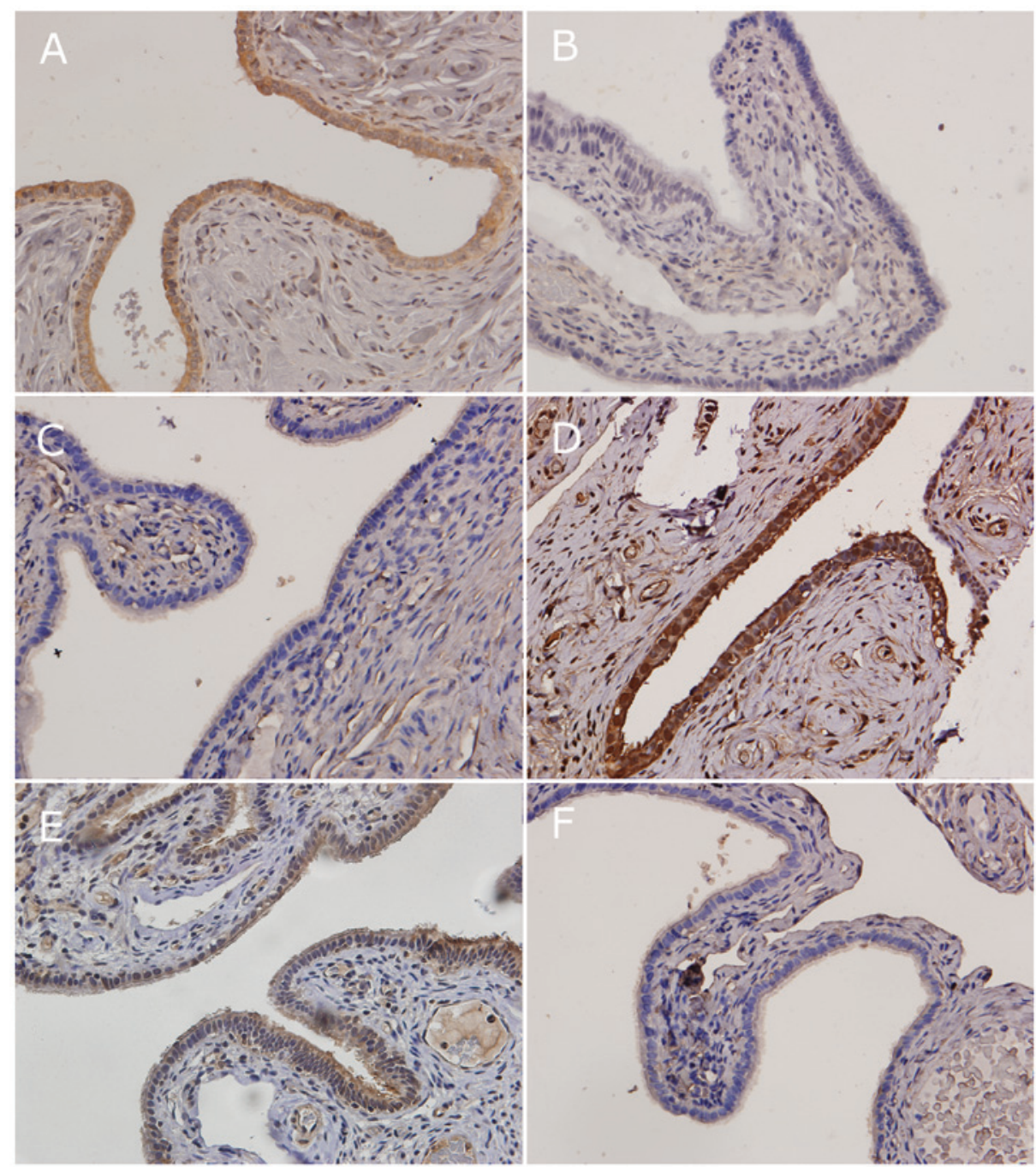

Figure 1. Cases of fimbrial epithelial cells in (A) high-grade and (B) low-grade OSC exhibiting strong nuclear positivity for pAKT. The high-grade lesions consistently showed more staining than the low-grade tumors. Cases of fimbrial epithelial cells in (C) high-grade and (D) low-grade OSC exhibiting strong nuclear positivity for E-cadherin. The high-grade lesions consistently showed less staining than the low-grade tumors. Cases of fimbrial epithelial cells in (E) high-grade and (F) low-grade OSC exhibiting strong nuclear positivity for COX-2. The high-grade lesions consistently showed more staining than the low-grade tumors. OSC, ovarian serous carcinoma; pAKT, phospho-AKT; COX-2, cyclooxygenase-2.

Table II. Immunohistochemical staining results for antibodies.

$$
\text { Positive staining, n (\%) }
$$

Biomarker High-grade tumors Low-grade tumors P-value

\begin{tabular}{lrcl}
\hline pAKT & $17 / 28(61)$ & $2 / 24(8)$ & 0.005 \\
MMP-2 & $6 / 28(21)$ & $3 / 24(13)$ & 0.78 \\
E-cadherin & $6 / 28(21)$ & $20 / 24(83)$ & 0.003 \\
VEGF & $7 / 28(25)$ & $5 / 24(21)$ & 0.86 \\
COX-2 & $20 / 28(71)$ & $5 / 24(21)$ & 0.007 \\
p53 & $4 / 28(14)$ & $2 / 24(8)$ & 0.82 \\
\hline
\end{tabular}

pAKT, phospho-AKT; COX-2, cyclooxygenase-2; MMP-2, matrix metalloproteinase-2; VEGF, vascular endothelial growth factor.

low- and high-grade OSCs, whereas the remaining 3 markers (MMP-2, VEGF and p53) had similar expression levels in both low- and high-grade OSCs.
The immunostaining levels of pAKT and COX-2 were significantly higher in the fimbriae of high-grade OSCs than those of low-grade OSCs. E-cadherin expression was significantly lower in the fimbriae of high-grade OSCs than those of low-grade OSCs (Fig. 1).

The results suggested important biological differences in the behavior of the fimbria in high- and low-grade OSCs and indicate that the proliferation, cell adhesion and inflammatory microenvironment of fimbriae of high-grade OSCs without STIC had changed prior to p53 mutation.

\section{Discussion}

According to Vaughan et al, ovarian cancer is many diseases (7). In the past few years, on the basis of a series of morphological and molecular genetic studies, a dualistic model has been proposed that categorizes various types of ovarian cancer into two groups, types I and II. The high-grade OSCs, which are the most common and most lethal of all ovarian epithelial neoplasms, may arise in the distal part of 
the Fallopian tubes (fimbria, secretory-type cells) and are only secondarily deposited on the ovarian surface. They commonly show genetic instability and p53 mutations (8).

However, a PubMed search revealed no earlier studies dealing with normal fimbriae of high- and low-grade OSCs and evaluating which characteristics had altered prior to p53 mutation. In this study, we evaluated the expression of 6 proteins in 28 cases of high-grade OSC and in 26 cases of low-grade OSC. In high-grade OSCs, there was a trend toward increased expression of pAKT and COX-2 in fimbriae without involvement of cancer. Higher E-cadherin positivity was observed in fimbriae of low-grade OSCs than in fimbriae of high-grade OSCs. This indicates that the proliferation, cell adhesion and inflammatory microenvironment of the fimbriae of high-grade OSCs without STIC had changed prior to p53 mutation.

An abundance of basic investigative studies has established the pAKT pathway as a major driver of cancerous behavior, inolved in proliferation, dysregulation of the cell cycle, apoptosis, metabolism, protein synthesis, senescence and other aspects of cell function. In addition, pAKT immunohistochemistry may be useful in the diagnosis and characterization of precancerous and intraepithelial lesions (9). We observed an increased AKT expression in $61 \%$ of high-grade OSCs, as compared with $8 \%$ of low-grade OSCs by immunohistochemistry.

E-cadherin is an adhesion molecule that may be involved in the metastasis of ovarian cancer (10). It has been suggested that E-cadherin acts as a tumor suppressor; furthermore, the transfection of tumor cells with E-cadherin cDNA prevents invasive growth (11). Thus, reduced cytoplasmic positivity of E-cadherin in high-grade OSC in this study is consistent with the poor outcome of patients with this disease. In our study, its expression was higher in the fimbriae of low-grade OSC than those of high-grade OSC. It is therefore possible that transformed fimbrial epithelial cells of high-grade OSC lose adhesion early in their progression and may slough off and migrate to the ovarian surface or directly to the peritoneum, with minimal ovarian deep involvement.

COX-2 overexpression has been reported in most gynecological neoplasms, including breast, cervix, endometrial and epithelial ovarian cancers. COX-2 expression promotes tumor cell proliferation, reduces apoptosis and induces angiogenesis (12). With regard to the inflammatory process, the cell proliferation rate has a well-known association with prognosis in ovarian carcinoma (13). The different expression levels of Cox-2 in low- and high-grade OSCs in our study possibly indicates the different inflammatory microenvironments.

The mechanism by which MMP-2 facilitates early adhesion and invasion involves cleavage of multiple extracellular matrix (ECM) proteins into smaller fragments that serve as better attachment sites. In addition, the inhibition of MMP-2, but not MMP-9, has been reported to significantly reduce ovarian cancer metastasis (14). A previous study detected active MMP-2 enzyme (62 kDa) only in ovarian cancer $(66 \%)$ and corresponding metastases $(93 \%)$, but never in benign or low potential malignancy tumors (15). We found that the expression level of MMP-2 was similar between the fimbriae of low- and high-grade OSCs, suggesting that the invasiveness of the fimbrial epithelial cells had not emerged.

VEGF expression in ovarian cancer has been evaluated in several studies. In early stage ovarian cancers, increased
VEGF expression has been shown to correlate with worse disease-free survival (DFS) and poor overall survival (OS) (16). In addition, a higher serum level of VEGF associated with ovarian cancer have been considered as an independent risk factor and a prognostic parameter for ascites, more metastasis, advanced-stage disease and reduced survival $(17,18)$. We have confirmed that the expression of VEGF had similar expression levels in low- and high-grade OSCs, maybe due to the alteration of fimbrial epithelial cells being an early event in the pathogenesis of high-grade OSCs and angiogenesis having not yet started.

p53 is a useful biomarker for detecting not only the early precursor lesions of high-grade OSCs, but also the later stages of this disease (19). Lee et al hypothesized that p53 signatures represent the elusive OSC precursor. In addition, short stretches of normal appearing Fallopian tube epithelium that strongly express p53, and in which p53 mutations have been identified in some cases, have been termed 'p53 signatures' (5). Although these lesions may represent early events in serous carcinogenesis, it is not clear, at this time, whether p53 signatures are precursor lesions or if they are benign 'reactive' changes that overexpress p53 and have no biological relevance to neoplasia. It has been shown that the expression of p53 mutations was similar between the fimbriae of normal appearance of low- and high-grade OSCs in our study. It is possible that p53 mutations were preceded by other neoplastic changes of cells, maybe after the increase of pAKT and COX-2 and the decrease of E-cadherin; this is an area that requires further investigation.

The present study has limitations, including its retrospective design, which is prone to selection bias, and a small sample size. In addition, the technique of immunohistochemistry does not always reflect the structure and functionality of the protein.

In conclusion, our results showed that the immunostaining of pAKT and COX-2 were significantly higher in the fimbriae of normal appearance of high-grade OSCs than those of low-grade OSCs and that the immunostaining of E-cadherin was significantly higher in the fimbriae of low-grade OSCs than those of high-grade OSCs. The remaining 3 markers (MMP-2, VEGF and p53) had similar expression levels in low- and highgrade OSCs. The relative importance of the Fallopian tube compared with the ovarian surface epithelium in the genesis of high-grade serous ovarian cancers is still being debated. However, our results suggest marked biological differences in the behavior of the fimbriae in high- and low-grade OSCs and indicate that the proliferation, cell adhesion and inflammatory microenvironment of fimbriae of high-grade OSCs without STIC had changed prior to p53 mutation.

\section{References}

1. Malpica A, Deavers MT, Lu K, et al: Grading ovarian serous carcinoma using a two-tier system. Am J Surg Pathol 28: 496-504, 2004.

2. Burks RT, Sherman ME and Kurman RJ: Micropapillary serous carcinoma of the ovary. A distinctive low-grade carcinoma related to serous borderline tumors. Am J Surg Pathol 20: 1319-1330, 1996.

3. Kurman RJ, Seidman JD and Shih IM: Serous borderline tumours of the ovary. Histopathology 47: 310-315, 2005.

4. Bowtell DD: The genesis and evolution of high-grade serous ovarian cancer. Nat Rev Cancer 10: 803-808, 2010. 
5. Lee Y, Miron A, Drapkin R, et al: A candidate precursor to serous carcinoma that originates in the distal fallopian tube. J Pathol 211: 26-35, 2007

6. Kindelberger DW, Lee Y, Miron A, et al: Intraepithelial carcinoma of the fimbria and pelvic serous carcinoma: Evidence for a causal relationship. Am J Surg Pathol 31: 161-169, 2007.

7. Vaughan S, Coward JI, Bast RC Jr, et al: Rethinking ovarian cancer: recommendations for improving outcomes. Nat Rev Cancer 10: 719-725, 2011.

8. Kurman RJ and Shih Ie-M: Molecular pathogenesis and extraovarian origin of epithelial ovarian cancer - shifting the paradigm. Hum Pathol 42: 918-931, 2011.

9. Shtilbans V, Wu M and Burstein DE: Current overview of the role of Akt in cancer studies via applied immunohistochemistry. Ann Diagn Pathol 12: 153-160, 2008.

10. Fujioka T, Takebayashi Y, Kihana T, et al: Expression of E-cadherin and beta-catenin in primary and peritoneal metastatic ovarian carcinoma. Oncol Rep 8: 249-255, 2001.

11. Vleminckx K, Vakaet L Jr, Mareel M, et al: Genetic manipulation of E-cadherin expression by epithelial tumor cells reveals an invasion suppressor role. Cell 66: 107-119, 1991.

12. Munkarah A and Ali-Fehmi R: COX-2: a protein with an active role in gynecological cancers. Curr Opin Obstet Gynecol 17: 49-53, 2005.
13. Kaern J, Aghmesheh M, Nesland JM, et al: Prognostic factors in ovarian carcinoma stage III patients. Can biomarkers improve the prediction of short- and long-term survivors? Int J Gynecol Cancer 15: 1014-1022, 2005.

14. Kenny HA and Lengyel E: MMP-2 functions as an early response protein in ovarian cancer metastasis. Cell Cycle 8: 683-688, 2009.

15. Schmalfeldt B, Prechtel D, Härtking K, et al: Increased expression of matrix metalloproteinases (MMP)-2, MMP-9, and the urokinase-type plasminogen activator is associated with progression from benign to advanced ovarian cancer. Clin Cancer Res 7: 2396-2404, 2001.

16. Paley P, Staskus K, Gebhard K, et al: Vascular endothelial growth factor expression in early stage ovarian carcinoma. Cancer 80: 98-106, 1997.

17. Cooper BC, Ritchie JM, Broghammer CL, et al: Preoperative serum vascular endothelial growth factor levels: significance in ovarian cancer. Clin Cancer Res 8: 3193-3197, 2002.

18. Hefler L, Zeillinger R, Grimm C, et al: Preoperative serum vascular endothelial growth factor as a prognostic parameter in ovarian cancer. Gynecol Oncol 103: 512-517, 2006.

19. Shih IeM and Kurman RJ: Ovarian tumorigenesis: a proposed model based on morphological and molecular genetic analysis. Am J Pathol 164: 1511-1518, 2004. 